\title{
The peculiar galaxy Mkn 298 revisited with integral field spectroscopy ${ }^{\star}$
}

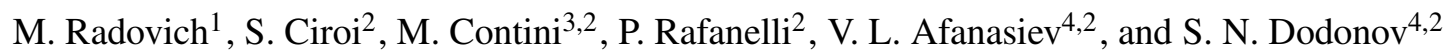 \\ ${ }^{1}$ INAF, Osservatorio Astronomico di Capodimonte, via Moiariello 16, 80131, Napoli, Italy \\ e-mail: radovich@na.astro.it \\ 2 Dipartimento di Astronomia, Università di Padova, Vicolo dell'Osservatorio 2, 35122 Padova, Italy \\ e-mail: [ciroi;piraf]@pd.astro.it \\ 3 School of Physics and Astronomy, Tel-Aviv University, Ramat-Aviv, Tel-Aviv, 69978 Israel \\ e-mail: contini@post.tau.ac.il \\ ${ }^{4}$ Special Astrophysical Observatory, Nizhnij Arkhyz, 369167 Russia \\ e-mail: [vafan;dodo]@sao.ru
}

Received 23 August 2004 / Accepted 4 October 2004

\begin{abstract}
Spectroscopic and imaging data of the peculiar galaxy Mkn 298 are presented. Narrow-band H $\alpha$ and broad-band $R$ images are used to study the star formation rate in the galaxy and its morphology, which is typical of a merging system. Long-slit and integral field spectra are used to assess the kinematics of gas and stars, and the nature of the ionizing source at different distances from the nucleus. In particular, the nucleus of Mkn 298 is characterized by peculiar line ratios: [N II] $] 6583 / \mathrm{H} \alpha$ is typical of $\mathrm{H}$ II-like regions, while [O I] $\lambda 6300 / \mathrm{H} \alpha$ could indicate the presence of an active galactic nucleus. We show that models where a shock component is added to photoionization from a starburst allow us to reproduce the observed line ratios. Mkn 298 is thus most likely a star-forming galaxy, rather than a galaxy hosting an active nucleus.
\end{abstract}

Key words. galaxies: peculiar - galaxies: individual: Mkn 298 - shock waves

\section{Introduction}

Mkn 298 (IC 1182) is known as a morphologically peculiar system (Fig. 1) located in the Hercules supercluster. It shows a chain (c, d, e) of small compact blue emitting regions (Stockton 1972) aligned on its eastern side up to $\sim 80^{\prime \prime}$ from the main body of the galaxy, which is classified in NED (NASA/IPAC Extragalactic Database) as a SA0+ galaxy. These regions are aligned with a tail (b) resembling a spiral arm located on the eastern side of the galaxy (a). On the western side a very faint trace of a spiral arm is also visible. A faint emission is visible in the c knot, behind a foreground star (Stockton 1972; Metik $\&$ Pronik 1982). The $\mathrm{d}$ and e knots are characterized by an emission line spectrum. They were identified by Braine et al. (2001) as a tidal dwarf galaxy (TDG hereafter) forming from material ejected from the disk of a galaxy after a collision.

The classification of Mkn 298 as a galaxy hosting an active nucleus is controversial in the literature. Koski (1978) and Veilleux \& Osterbrock (1987) classified it as a Seyfert 2 galaxy with an extremely low [N II] $\lambda 6583 / \mathrm{H} \alpha$ line ratio. Conversely Viegas-Aldrovandi \& Gruenwald (1990) included Mkn 298

* Based on data obtained at the $2.2 \mathrm{~m}$ and NTT telescopes of ESOla Silla (Chile), at the $2.2 \mathrm{~m}$ telescope of DSAZ-Calar Alto (Spain), and at the $6 \mathrm{~m}$ telescope of SAO (Russia).

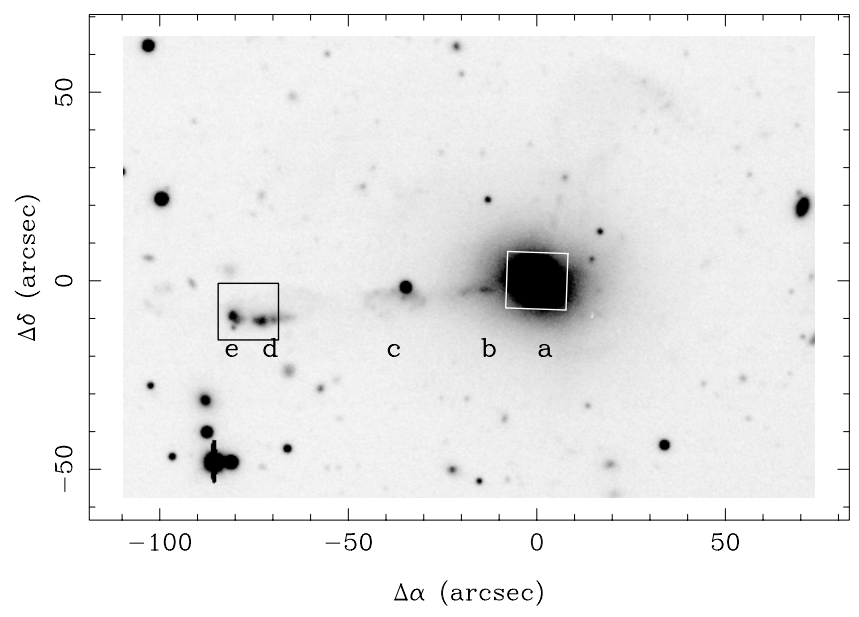

Fig. 1. The Mkn 298 complex in the $R$-band image with the position of the MPFS arrays overlaid.

in a sample of LINERs. Variability was claimed by Metik \& Pronik (1982) who observed changes in the relative intensity of [O III] $\lambda \lambda 4959,5007$ and $\mathrm{H} \beta$ in the period 1969-70 and 1977. The detection of variable soft X-ray emission (Rafanelli et al. 1999) with a luminosity typical of Seyfert 2 galaxies seems to confirm the presence of an active nucleus. However, as noted 
by Moles et al. (2004) it is not possible to exclude that the $\mathrm{X}$-ray emission is produced in star-forming regions (Zezas et al. 2002). Moles et al. (2004) detected the presence of several knots in the nucleus; they interpreted Mkn 298 as a very luminous starburst $\left(S F R \sim 90 M_{\odot} / \mathrm{yr}\right)$ with very low metallicity $\left(Z / Z_{\odot} \sim 0.06-0.1\right)$.

An extended H I distribution peaked on the TDG was detected using VLA data (Braine et al. 2001; Iglesias-Páramo et al. 2003). Braine et al. (2001) report the detection of $\mathrm{CO}(2-1)$ and $\mathrm{CO}(1-0)$ emission in Mkn 298, however the emission peaks at the main galaxy and is below detection in the TDG. They computed a total amount of $\mathrm{H}_{2}$ mass in the system $M_{\text {mol }}>5 \times 10^{9} M_{\odot}$, and an upper limit $M_{\text {mol }}<6 \times 10^{7} M_{\odot}$ in the TDG. This would imply that only a small fraction of H I was transformed into $\mathrm{H}_{2}$ in the TDG, while in the nucleus the conversion had already taken place. This is consistent with Mkn 298 being in the late stage of merging: as outlined by Hibbard \& van Gorkom (1996), in advanced mergers the atomic gas is mostly relegated to the outer tidal features and is almost absent in the central regions, whereas the molecular gas is concentrated in the remnant body.

The aim of this paper is to study in detail the physical conditions in the different components (galaxy body and tail) of the Mkn 298 system by the analysis of their emission-line spectra. Observations and data reduction steps are described in Sect. 2. The morphology, star formation rates and kinematics are analyzed and discussed in Sects. 3-5 respectively. In Sect. 6 emission line ratios are used to derive chemical abundances. Observed ratios are then compared with the results from both pure photoionization models and photoionization+shock models, using the CLOUDY and SUMA codes respectively. Conclusions are drawn in Sect. 7.

\section{Observations and data reduction}

Emission line spectra and images of Mkn 298 have been taken in different periods and using different observing facilities.

\subsection{Imaging}

1. Narrow band images in $\mathrm{H} \alpha\left(\lambda_{\mathrm{c}}=6820 \AA, \Delta \lambda=170 \AA\right)$ and continuum light $\left(\lambda_{\mathrm{c}}=6240 \AA, \Delta \lambda=110 \AA\right)$ were taken with the Deutsch-Spanische Astronomische Zentrum of Calar Alto (DSAZ) $2.2 \mathrm{~m}$ telescope (Spain) in September 1996, with a $2 \mathrm{k} \times 2 \mathrm{k}$ CCD (spatial scale $0.53^{\prime \prime} / \mathrm{px}$ ), and under a seeing of $\sim 1.5^{\prime \prime}$. Images of the galaxy of 600 and $900 \mathrm{~s}$ were obtained with the two filters, respectively. Moreover, the spectrophotometric standard star LDS749b was observed for flux calibration. All images were processed by means of the IRAF $^{1}$ packages. They were firstly bias subtracted, flatfielded and cosmic-ray cleaned. Then, we performed the photometry of the standard star, whose fluxes

${ }^{1}$ IRAF is distributed by the National Optical Astronomy Observatories, which are operated by the Association of Universities for Research in Astronomy, Inc., under cooperative agreement with the National Science Foundation. were measured with PHOT, corrected for atmospheric extinction and compared to the fluxes obtained from the spectrum of the star convolved with the response curve of the filters to obtain the calibration constants. The filter transmissions were taken into account. The images of the galaxy were aligned, background subtracted, normalized to $1 \mathrm{~s}$ exposure and corrected for atmospheric extinction. Then, the continuum image was scaled by a factor of 1.6 to compensate for the different shapes of the two filters. The respective calibration constants were applied to the processed images, and finally the continuum was subtracted from $\mathrm{H} \alpha$ to obtain a pure emission line image.

2. Five consecutive broad-band $R$ exposures of Mkn 298, 15 min each, were obtained with EMMI at the ESO-NTT in August 1991 . The $2 \mathrm{k} \times 2 \mathrm{k}$ CCD, with $15 \mu$ pixels, yielded a spatial scale of $0.348^{\prime \prime} / \mathrm{px}$. The seeing was $1.4^{\prime \prime}$. The five images were bias and flat-field corrected, then aligned and combined to obtain an average frame cleaned of cosmic rays. No photometric calibration was performed.

3. A public image of the galaxy was extracted from the HST Archive. Obtained in June 1995 with the WFPC2 in combination with the $F 606 W$ filter, and 500 s of exposure time (see Malkan et al. 1998), this image was simply cleaned of cosmic-rays and directly analyzed. The main part of the galaxy was covered with the PC1, having a spatial scale of $0.045^{\prime \prime} / \mathrm{px}$.

\subsection{Spectroscopy}

1. The nucleus was observed in May and June 1999 with the Multi Pupil Fiber Spectrograph (MPFS) at the $6 \mathrm{~m}$ telescope of the Special Astrophysical Observatory (SAO RAS, Russia). The MPFS is an integral field spectrograph, in which an array $(16 \times 15$ at the epoch of the observations) of $1^{\prime \prime} \times 1^{\prime \prime}$ microlenses is connected to a bundle of fiber optics. The 240 fibers are re-arranged to form a pseudoslit which is the input to the spectrograph. A $600 / \mathrm{mm}$ grating was used during the first run (R1) of observations, in combination with a $1 \mathrm{k} \times 1 \mathrm{k}$ CCD. Each of the two spectral ranges, 3350-6100 $\AA$ and 4550-7300 , was observed with a dispersion of $\sim 2.6 \AA / \mathrm{px}$, an instrumental $F W H M$ of $7.5 \AA\left(\sim 400 \mathrm{~km} \mathrm{~s}^{-1}\right.$ at $\left.5500 \AA\right)$ and an exposure time of $3600 \mathrm{~s}$. During the second run (R2) a 1200/mm grating was used, which covered the ranges 4800-6200 $\AA$ and 5900-7300 $\AA$, with a dispersion of $\sim 1.36 \AA / \mathrm{px}$ and an instrumental $F W H M$ of $4.5 \AA\left(\sim 250 \mathrm{~km} \mathrm{~s}^{-1}\right.$ at $\left.5500 \AA\right)$. Integration times of $7200 \mathrm{~s}$ for the "blue spectrum" and $4800 \mathrm{~s}$ for the "red spectrum" were applied. The seeing was typically around $1.5-2$ " for both runs.

The MPFS spectra were reduced and flux calibrated with a special software developed in IDL at SAO.

2. Knots d, e were also observed with the MPFS during the above runs. In this case, a $300 / \mathrm{mm}$ grating was used, which allowed us to cover the whole optical spectral range 3600-9000 ̊, with an instrumental $F W H M \sim 15 \AA$. An exposure time of $3600 \mathrm{~s}$ was used. Long-slit spectra of the same knots were taken with EFOSC2 at the 2.2-m telescope 

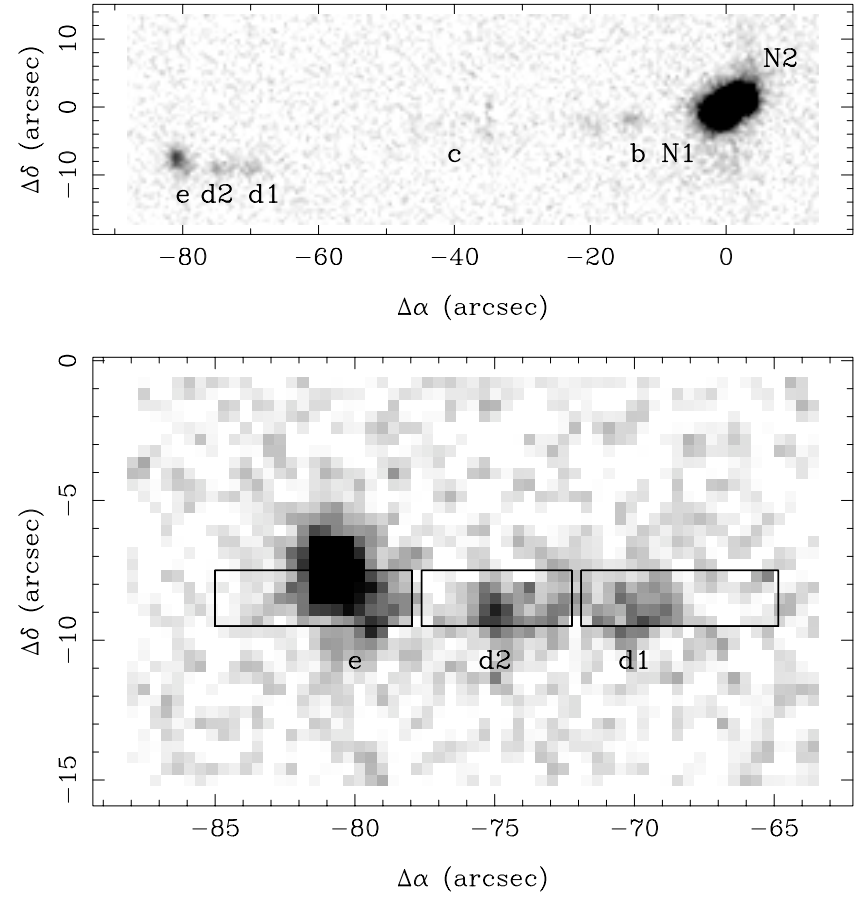

Fig. 2. Continuum-subtracted $\mathrm{H} \alpha$ image of Mkn 298 with the position of the ESO slit overlaid on the d, e knots.

at ESO-La Silla (Chile) in April 1995. The instrumental $F W H M$ is $\sim 10 \AA$, the covered range is $4500-7000 \AA$. The standard data reduction steps (bias subtraction, flatfield correction and flux calibration) were applied using the LONGSLIT package in IRAF. Due to the higher spectral resolution of the ESO spectra, we decided to use them rather than the MPFS data in the analysis of the knots, with the exception of the [O II] $\lambda 3727$ line which is covered by the MPFS spectra only.

The positions of the slits and MPFS spectra are displayed in Figs. 1 and 2, where they are overplotted on the $R$-band and $\mathrm{H} \alpha$ images of Mkn 298 respectively. The $\mathrm{H} \alpha$ image clearly shows the existence of two knots in the nucleus which were further resolved into four knots (CK, CKN1 and CKN2, CKN4) by Moles et al. (2004). The knots are not visible in the $R$-band image.

A $2^{\prime \prime} \times 2^{\prime \prime}$ rebinning of the MPFS spectra was done before the measurements in order to increase the signal to noise ratio: each measured spectrum is therefore the sum of four MPFS spectra. Spectra from R2 were used to obtain a 2D map of the emission line ratios with the highest spectral resolution. Finally, two regions corresponding to the nuclear knots (N1, N2) were defined and spectra inside them were summed. In this case, spectra from $\mathrm{R} 1$ were used to cover the full spectral range from [O II] $\lambda 3727$ to [S II] $\lambda 6716,31$, but with a lower spectral resolution.

Emission line ratios after correction for reddening are shown in Table 2. For each region, we selected the spectra with the best spectral resolution, namely the MPFS spectra for the nucleus and the ESO spectra for the d, e knots. Uncertainties on the measured line ratios are $\leq 10 \%$ in the nucleus, $\sim 20-30 \%$ in the knots. The uncertainty is much higher $(\sim 50 \%)$ in the case

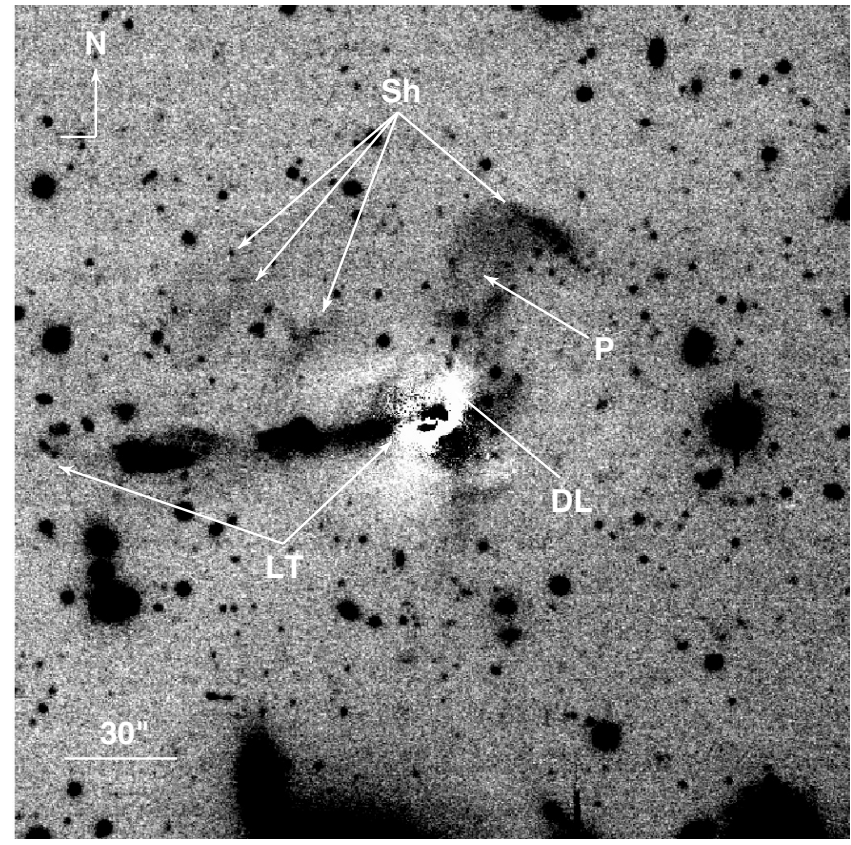

Fig. 3. Residuals after the GIM2D two-dimensional model subtraction: white arrows indicate the long tail (LT), the dust lane (DL), the plume (P) and the shells (Sh).

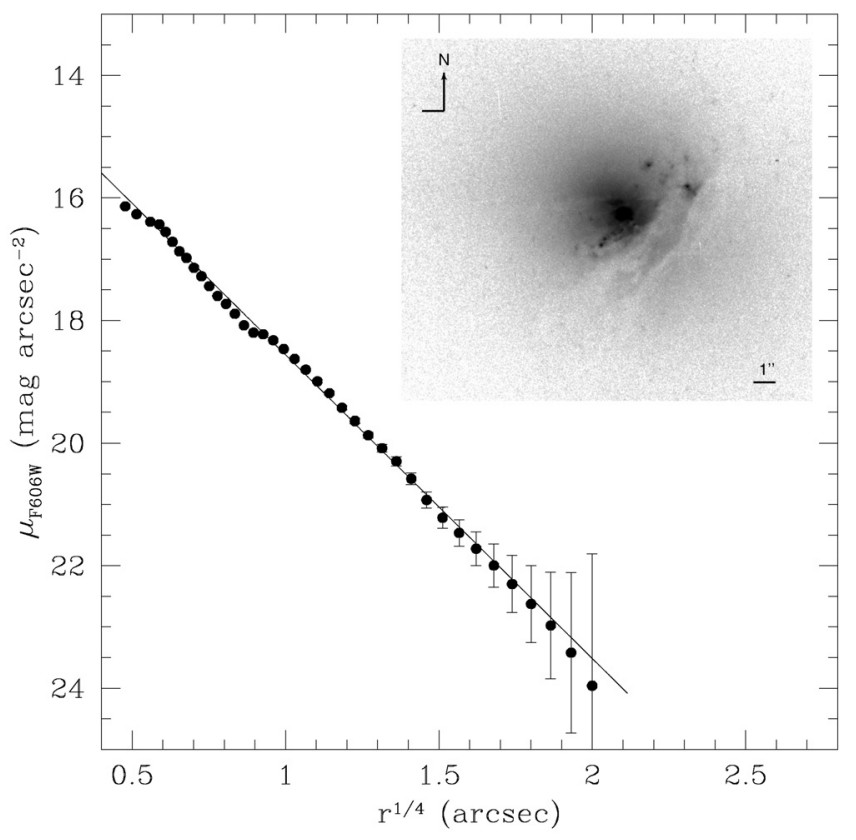

Fig. 4. HST image of Mkn 298 and fit of the surface brightness profile.

of [O III] $\lambda 4363$, due both to the nearby $\mathrm{H} \gamma \lambda 4340$ and to the noise in the continuum.

The redshift of Mkn 298 as derived from the peak positions of the lines is $z=0.034$, giving a linear scale of $0.66 \mathrm{kpc} / \mathrm{arcsec}$ $\left(H_{0}=75 \mathrm{~km} \mathrm{~s}^{-1} \mathrm{Mpc}^{-1}\right)$.

\section{Morphology}

Understanding the peculiar morphology of Mkn 298 requires a detailed analysis of deep images. For this purpose we applied the GIM2D package (Simard et al. 2002) to the NTT 
$R$-band image, which performs a two-dimensional bulge/disk decomposition of galaxy images, and produces as output the model convolved with the PSF and the residual image (=galaxy-model). Mkn 298 was first fitted using a Sersic $R^{1 / n_{-}}$ law for the bulge and a classical exponential law for the disk. The fit gave as result an index $n=3.5$ and a reduced- $\chi^{2} \sim 1.28$. Then a deVaucouleurs $R^{1 / 4}$-law was applied for the bulge obtaining quite similar residuals (reduced- $\chi^{2} \sim 1.16$ ). The resulting $\mathrm{B} / \mathrm{T}$ ratio $\sim 0.78$ indicates clearly that $\mathrm{Mkn} 298$ is a spheroidal galaxy, likely an S0, with a weak disk inclined at $i \sim 40^{\circ}$. The fit also gave the effective radius of the bulge, $r_{\mathrm{e}} \sim 7^{\prime \prime}$ and the radius $r_{\text {half }} \sim 9.5^{\prime \prime}$ containing half of the total integrated light.

The structure and morphology of the ionized gas in the nucleus was discussed in detail by Moles et al. (2004). In addition, we note that the strong nuclear dust lane, whose maximum radial extension is $\sim 17^{\prime \prime}(\sim 11 \mathrm{kpc})$, leaves a residual resembling a ring inclined at $i \sim 140-145^{\circ}$. Interestingly, the galaxy shows at least three shells located at $\sim 40^{\prime \prime}, 1^{\prime}$ and $1.3^{\prime}$ ( $\sim 26,42$ and $50 \mathrm{kpc}$ respectively) East and North-East of the nucleus, and another one at $\sim 58^{\prime \prime}(\sim 38 \mathrm{kpc})$ North-West of the nucleus, connected to a large plume oriented at PA $\sim 160^{\circ}$, and named the Second Tail by Moles et al. (2004) (see Fig. 3). These features are not uncommon in elliptical and S0 galaxies, and are still studied to understand their real nature and origin. The simulations by Hernquist \& Spergel (1992) show that mergers between equal-mass galaxies can develop such shells on timescales of about 1-2 Gyr. Turnbull et al. (1999) studied two examples of shell ellipticals, which have very strong analogies with those present in Mkn 298 (see their Figs. 9 and 10). Nevertheless, Mkn 298 is a rare example of an earlytype galaxy showing both shells and a prominent stellar and gaseous tidal tail, a situation not seen in the above-mentioned numerical simulations. Faint tidal tails were found by Balcells (1997) in the shell elliptical NGC 3656, and several shells and a long $\mathrm{H}$ I tidal tail were discovered by Verdes-Montenegro et al. (2002) in HCG 54. Only the NW shell has a surface brightness close to $3 \sigma$ above the background. Assuming a sky brightness $\mu_{R} \sim 20.5 \mathrm{mag} \operatorname{arcsec}^{-2}$, the value expected at LaSilla for similar moon illumination conditions, we obtain $\mu_{R} \sim 25 \mathrm{mag} \mathrm{arcsec}^{-2}$ : such a value is in agreement with those measured by Turnbull et al. for the shells of their galaxies. The other shells in Mkn 298 have surface brightnesses between 1 and $2 \sigma$ of the background. In the residual image it can be also seen that the Eastern tidal tail extends beyond the $\mathrm{d}$ and e knots, up to $1.75^{\prime}(\sim 70 \mathrm{kpc})$, where it seems to turn back towards the North-West.

The nucleus of Mkn 298 was also observed by HST with the WFPC2 and the broad-band $V$ filter F606W (Malkan et al. 1998). An analysis of the isophotes of the galaxy was done using the ELLIPSE task in IRAF. Since the presence of the dust lane makes it difficult to fit the real isophotes, we applied the masking method used by Carollo et al. (1997). The surface brightness profile plotted against $r^{1 / 4}$ can be fitted by a de Vaucouleurs law $\left(r_{\mathrm{e}} \sim 8^{\prime \prime}\right)$ without an additional disk component; it does not show any nuclear point-like sources (e.g. AGN or stellar cluster), in agreement with the results given by GIM2D.
Table 1. H $\alpha$ luminosities and star formation rates. The labels in parentheses are those adopted by Moles et al. (2004).

\begin{tabular}{lcccc}
\hline \hline & \multicolumn{2}{c}{ Observed } & \multicolumn{2}{c}{ Dereddened } \\
& $\log L_{\mathrm{H} \alpha}$ & SFR & $\log L_{\mathrm{H} \alpha}$ & $S F R$ \\
\hline N1 (CK+CKN1) & 41.46 & 2.30 & 41.83 & 5.35 \\
N2 (CKN2+CKN4) & 41.21 & 1.29 & 41.50 & 2.52 \\
a (galaxy body) & 41.72 & 4.19 & 42.01 & 8.14 \\
b (MTK1) & 40.02 & 0.08 & & \\
c (MTK3) & $\leq 39.87$ & $\leq 0.06$ & & \\
d1 (MTK4) & 39.93 & 0.07 & 40.52 & 0.26 \\
d2 (MTK5) & 40.03 & 0.08 & 40.58 & 0.30 \\
e (MTK6) & 40.42 & 0.21 & 40.42 & 0.21 \\
\hline
\end{tabular}

In summary, the brightest part of Mkn 298, that is within $\sim 6.3 \mathrm{kpc}$, is clearly spheroidal. Such a morphology, combined with the presence of a dust lane, a bright and extended tail and some weak shells, are all strong clues in favor of the hypothesis that Mkn 298 has experienced a major merger event.

\section{Star formation rates}

The presence of multiple knots in the nucleus is also visible in the continuum subtracted $\mathrm{H} \alpha$ image. Aperture photometry was done on the two brightest nuclear knots $(\mathrm{N} 1, \mathrm{~N} 2)$ and on the regions in the tail (b, c, d, e). A very faint $\mathrm{H} \alpha$ emission is detected in the $\mathrm{c}$ knot.

An estimate of the photometric accuracy was done by comparing the so-obtained fluxes with those obtained from the MPFS spectra. We conclude that the photometry of imaging and spectroscopic data is consistent within $5 \%$.

$\mathrm{H} \alpha$ luminosities obtained in each of these regions are shown in Table 1; for those regions where dust extinction from $\mathrm{H} \alpha / \mathrm{H} \beta$ was derived from spectra, the extinction-corrected values are also displayed. $\mathrm{H} \alpha$ luminosities were finally converted to star formation rates using Eq. (2) from Kennicutt (1998). In the nucleus, this is an upper limit if part of the $\mathrm{H} \alpha$ emission is due to an AGN. We see that the total star formation rate ( $a$ region) is $\sim 8.1 M_{\odot} \mathrm{yr}^{-1}$, well below the value of $90 M_{\odot} \mathrm{yr}^{-1}$ given by Moles et al. (2004). The $\mathrm{H} \alpha$ luminosities in the galaxy measured by us and by Moles et al. (2004) before correction for dust reddening are consistent within $30 \%$. The difference in the SFR is mainly due to the internal reddening adopted by Moles et al. (2004), $E(B-V) \sim 1$, which is much higher than the average reddening that we find in the nucleus, $E(B-V) \sim 0.3$. This value of the reddening is also in agreement with the value found by Koski (1978), $E(B-V)=0.40$. A possible reason for this discrepancy may be that the spectra that Moles et al. (2004) used to derive the extinction come from knots (CK and CKN2) which are very close to the dust lane: they could therefore be biased towards high values of $\mathrm{H} \alpha / \mathrm{H} \beta$, not representative of the whole galaxy.

Mkn 298 is not a bright IRAS source; the IRAS Faint Source Catalog contains an entry which is however centered on the d, e knots. Approximate fluxes at the position of Mkn 298 
Table 2. Dereddened emission line ratios $(\mathrm{H} \beta=1.00)$ and models computed assuming (a) photoionization only and (b) photoionization + shock (see text for model parameters). The values in italics are the uncertainties on the observed line ratios; the $\mathrm{H} \alpha / \mathrm{H} \beta$ ratio before reddening correction is also given below.

\begin{tabular}{|c|c|c|c|c|c|c|c|c|c|c|c|c|}
\hline & \multirow{2}{*}{$\begin{array}{c}\text { MPFS } \\
\text { N1 }\end{array}$} & \multirow{2}{*}{$\begin{array}{c}\text { MPFS } \\
\text { N2 }\end{array}$} & \multirow{2}{*}{$\begin{array}{c}\text { ESO } \\
\mathrm{d} 11\end{array}$} & \multirow{2}{*}{$\begin{array}{c}\text { ESO } \\
\mathrm{d} 2\end{array}$} & \multirow{2}{*}{$\begin{array}{c}\text { ESO } \\
\mathrm{e}\end{array}$} & \multicolumn{2}{|c|}{ Cloudy } & \multicolumn{2}{|c|}{$\mathrm{AGN}+$ shock } & \multicolumn{3}{|c|}{$\mathrm{SB}+$ shock } \\
\hline & & & & & & Nucleus & $\mathrm{d}, \mathrm{e}$ & $\mathrm{N} 1$ & $\mathrm{~N} 2$ & N1 & $\mathrm{N} 2$ & $\mathrm{~d}, \mathrm{e}$ \\
\hline \multirow[t]{2}{*}{ [O II] $\lambda 3727$} & 5.3 & 6.5 & & & $3^{1}$ & 6.98 & 3.92 & 6.00 & 4.90 & 5.30 & 5.70 & 3 \\
\hline & 0.1 & 0.3 & & & 1 & & & & & & & \\
\hline \multirow[t]{2}{*}{$\mathrm{Ne}$ III] $\lambda 3869$} & 0.20 & 0.2 & & & & 0.64 & 0.24 & 0.20 & 0.20 & 0.28 & 0.28 & 0.57 \\
\hline & 0.04 & 0.1 & & & & & & & & & & \\
\hline \multirow[t]{2}{*}{$\mathrm{H} \gamma \lambda 4340$} & 0.56 & 0.65 & & & & 0.47 & 0.47 & 0.44 & 0.44 & 0.46 & 0.46 & 0.46 \\
\hline & 0.04 & 0.05 & & & & & & & & & & \\
\hline \multirow[t]{2}{*}{ [O III] $\lambda 4363$} & 0.03: & 0.08 : & & & & 0.02 & 0.02 & 0.19 & 0.16 & 0.03 & 0.02 & 0.02 \\
\hline & 0.02 & 0.03 & & & & & & & & & & \\
\hline \multirow[t]{2}{*}{ [O III] $\lambda 4959$} & 0.67 & 0.70 & 0.7 & 1.9 & 0.8 & 0.95 & 1.01 & 0.58 & 0.50 & 0.67 & 0.54 & 0.95 \\
\hline & 0.03 & 0.04 & 0.5 & 0.5 & 0.1 & & & & & & & \\
\hline \multirow[t]{2}{*}{ [O III] $\lambda 5007$} & 1.90 & 1.98 & 3 & 2.9 & 2.7 & 2.85 & 3.02 & 1.73 & 1.50 & 2.00 & 1.62 & 2.85 \\
\hline & 0.06 & 0.08 & 1 & 0.7 & 0.3 & & & & & & & \\
\hline \multirow[t]{2}{*}{ [O I] $\lambda 6300$} & 0.35 & 0.40 & $<0.1$ & $<0.1$ & $\leq 0.1$ & 0.46 & 0.06 & 0.30 & 0.33 & 0.30 & 0.35 & 0.14 \\
\hline & 0.02 & 0.02 & & & & & & & & & & \\
\hline $\mathrm{H} \alpha \lambda 6563$ & 2.80 & 2.80 & 2.80 & 2.80 & 2.80 & 2.91 & 2.87 & 3.30 & 3.40 & 2.97 & 3.00 & 2.93 \\
\hline \multirow[t]{2}{*}{ [N II] $\lambda 6583$} & 0.86 & 0.65 & 0.4 & 0.3 & 0.22 & 0.92 & 0.23 & 0.80 & 0.90 & 0.82 & 0.93 & 0.33 \\
\hline & 0.03 & 0.03 & 0.2 & 0.1 & 0.05 & & & & & & & \\
\hline \multirow[t]{2}{*}{ [S II] $\lambda 6716$} & 0.72 & 0.83 & & & 0.32 & 1.24 & 0.30 & 0.80 & 0.86 & 0.72 & 0.81 & 0.37 \\
\hline & 0.03 & 0.03 & & & 0.05 & & & & & & & \\
\hline \multirow[t]{2}{*}{ [S II] $\lambda 6731$} & 0.54 & 0.51 & & & 0.30 & 0.92 & 0.40 & 0.67 & 0.82 & 0.60 & 0.76 & 0.29 \\
\hline & 0.02 & 0.02 & & & 0.05 & & & & & & & \\
\hline \multirow[t]{2}{*}{ [S II] $\lambda 6716+31$} & 1.26 & 1.34 & 0.9 & 0.7 & 0.6 & 2.16 & 0.70 & 1.47 & 1.68 & 1.32 & 1.57 & 0.66 \\
\hline & 0.05 & 0.05 & 0.3 & 0.2 & 0.1 & & & & & & & \\
\hline \multirow[t]{2}{*}{$\mathrm{H} \alpha \lambda 6563$} & 4.3 & 4.2 & 6 & 5 & 2.8 & & & & & & & \\
\hline & 0.1 & 0.1 & 2 & 1 & 0.2 & & & & & & & \\
\hline$E(B-V)$ & 0.40 & 0.37 & 0.61 & 0.57 & 0.00 & & & & & & & \\
\hline
\end{tabular}

${ }^{1}$ From MPFS data.

can be derived from the Iras Sky Survey Atlas using the IRSA ${ }^{2}$ facility: we obtain $f_{60 \mu \mathrm{m}}=0.8 \mathrm{Jy}, f_{100 \mu \mathrm{m}}=2.1 \mathrm{Jy}$. These values are very uncertain as the emission is distributed on a wide area ( $>2$ arcmin around the galaxy) and is probably the sum of the emission from the galaxy and from the tidal tail. According to Eqs. (7), (8) from Hopkins et al. (2003), we obtain $L(8-1000 \mu \mathrm{m})=1.8 \times 10^{44} \mathrm{erg} \mathrm{s}^{-1}$ and a $S F R \sim 11 M_{\odot} \mathrm{yr}^{-1}$, a value in good agreement with the SFR derived from the total $\mathrm{H} \alpha$ luminosity (galaxy+tidal tail). As the contribution to the SFR from the tidal tail estimated from the $\mathrm{H} \alpha$ luminosity is negligible compared to that in the galaxy, it seems likely that this is also the case for the infrared luminosity.

Georgakakis et al. (2000) explored the possibility of using the $L_{\mathrm{FIR}} / M\left(\mathrm{H}_{2}\right)$ ratio (star formation efficiency, SFE hereafter) as an indicator of the galaxy merging age. In the case of Mkn 298, we obtain SFE $\leq 9 L_{\odot} / M_{\odot}$ using the above farinfrared luminosity and the value $M_{\mathrm{mol}} \sim 5 \times 10^{9} M_{\odot}$ from Braine et al. (2001). Together with the low fraction of $\mathrm{HI}$ in the

\footnotetext{
${ }^{2}$ NASA/IPAC Infrared Science Archive.
}

nucleus, this value would indicate that Mkn 298 is in a postmerger stage. The $\mathrm{N} 1$ and $\mathrm{N} 2$ knots in the nucleus are probably separate star forming regions partially obscured by the dust lane, rather than separate nuclei before merging.

\section{Kinematics}

Taking advantage of the integral field data, we carried out a two-dimensional investigation of the gaseous and stellar kinematics in Mkn 298.

First, the wavelength positions of the two brightest emission lines $[\mathrm{O}$ III $] \lambda 5007$ and $\mathrm{H} \alpha$ were measured by Gaussian fitting of their profiles in each spectrum where they could be detected down to $3 \sigma$ over the continuum. Then, the $\operatorname{Mg} b \lambda 5175$ absorption triplet was chosen to derive the stellar kinematics, and the Fourier cross-correlation method was applied by means of the IRAF task FXCOR (Tonry \& Davis 1979, TD79 hereafter), and using a mean sky-flat spectrum as template. Only the correlation peaks with the TD79 parameter $R>5$, which is an indicator of the signal-to-noise ratio, were considered 

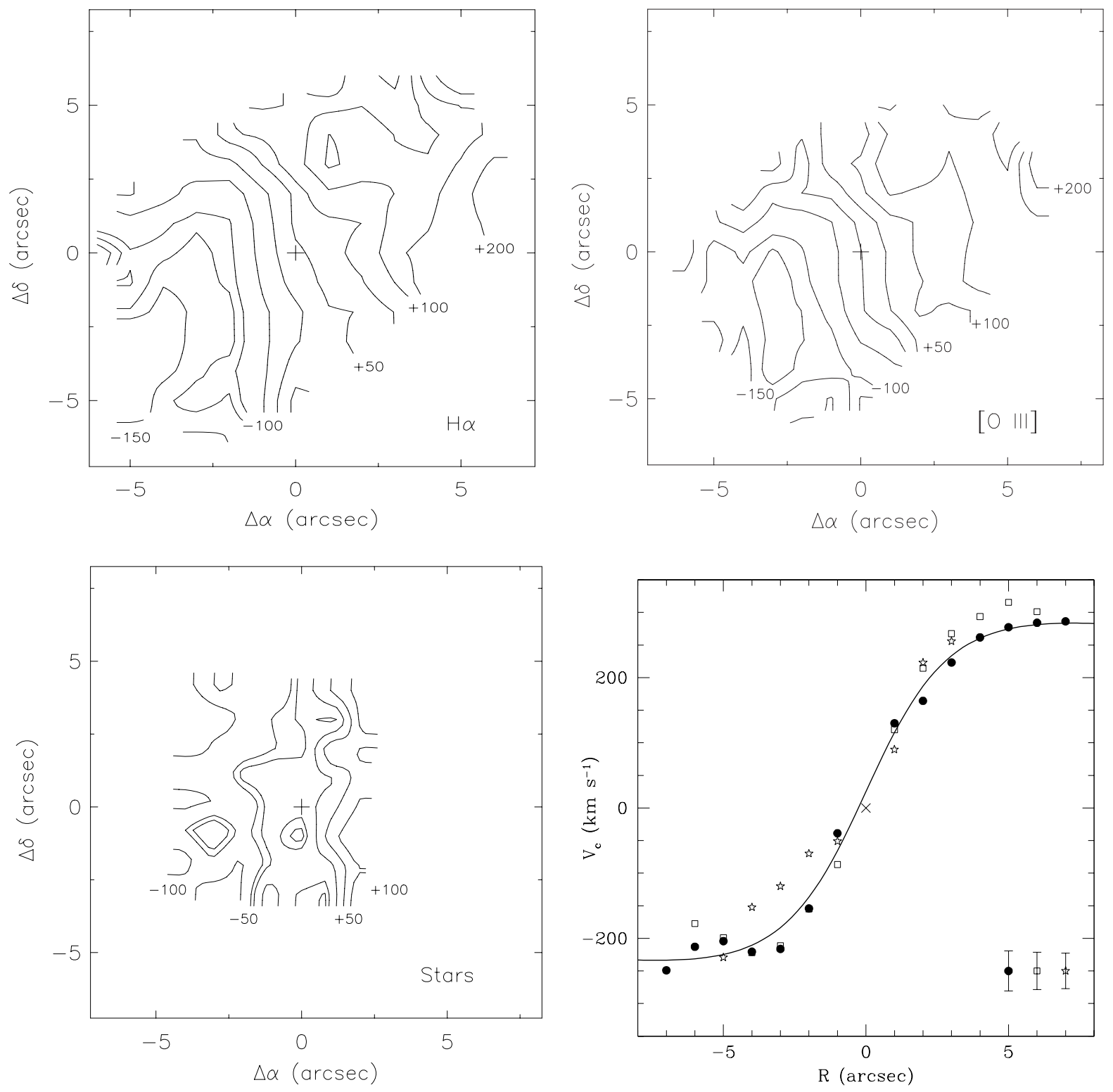

Fig. 5. Velocity fields of $\mathrm{H} \alpha$ (up-left), [O III] $\lambda 5007$ (up-right), and stars (bottom-left); the cross indicates the position of the nuclear continuum. North is up and East to the left. (bottom-right) The deprojected velocity curves of H $\alpha$ (filled circles), [O III] $\lambda 5007$ (open squares) and stars (open stars). The solid line is the pure circular motion fit of the gaseous component.

reliable. The so-obtained radial velocities were corrected for the motion of the observer in the direction of the observation, and then re-organized to construct the velocity fields for each component (Fig. 5). A first look reveals that the [O III] and $\mathrm{H} \alpha$ kinematics are in agreement, showing similar displacement of their "spider" diagrams, which are extended up to a maximum radius of $\sim 7^{\prime \prime}(\sim 4.6 \mathrm{kpc})$, and having concordant velocity values. In addition, their velocity fields have the major axes clearly parallel to the dust lane, strongly suggesting that the gas and the dust lane belong to the same structure, a rotating disk of a likely former spiral galaxy merged into an early-type or a gas-poor galaxy. On the contrary, stars show a velocity field with a slightly different orientation, indicating the presence of a kinematical decoupling.

In order to determine the rotation curve of the galaxy in the field of view of the spectrograph, we applied the GIPSY
(Groningen Image Processing SYstem) task ROTCUR. Based on the method described by Begeman (1989), ROTCUR derives kinematical parameters by fitting the velocity field with concentric tilted-rings, characterized by seven free parameters. In our case, given the relatively small number of available measurements, it was necessary to conveniently reduce the degrees of freedom. We followed the recipes by Begeman (1989) to determine the $X_{0}$ and $Y_{0}$ coordinates of the center, and the systemic velocity, $V_{\text {sys }} \sim 10250 \mathrm{~km} \mathrm{~s}^{-1}$, and in addition we fixed the inclination, $i$, of the rings on the basis of the optical isophotes. Therefore, ROTCUR fitted the circular velocity, $V_{\mathrm{c}}$, for increasing radii, varying the position angles of the rings. We performed this procedure separately for the approaching and the receding sides of the velocity field, in order to account for possible distortions or asymmetries. The resulting $V_{\mathrm{c}}(R)$ values were plotted to visualize the deprojected 


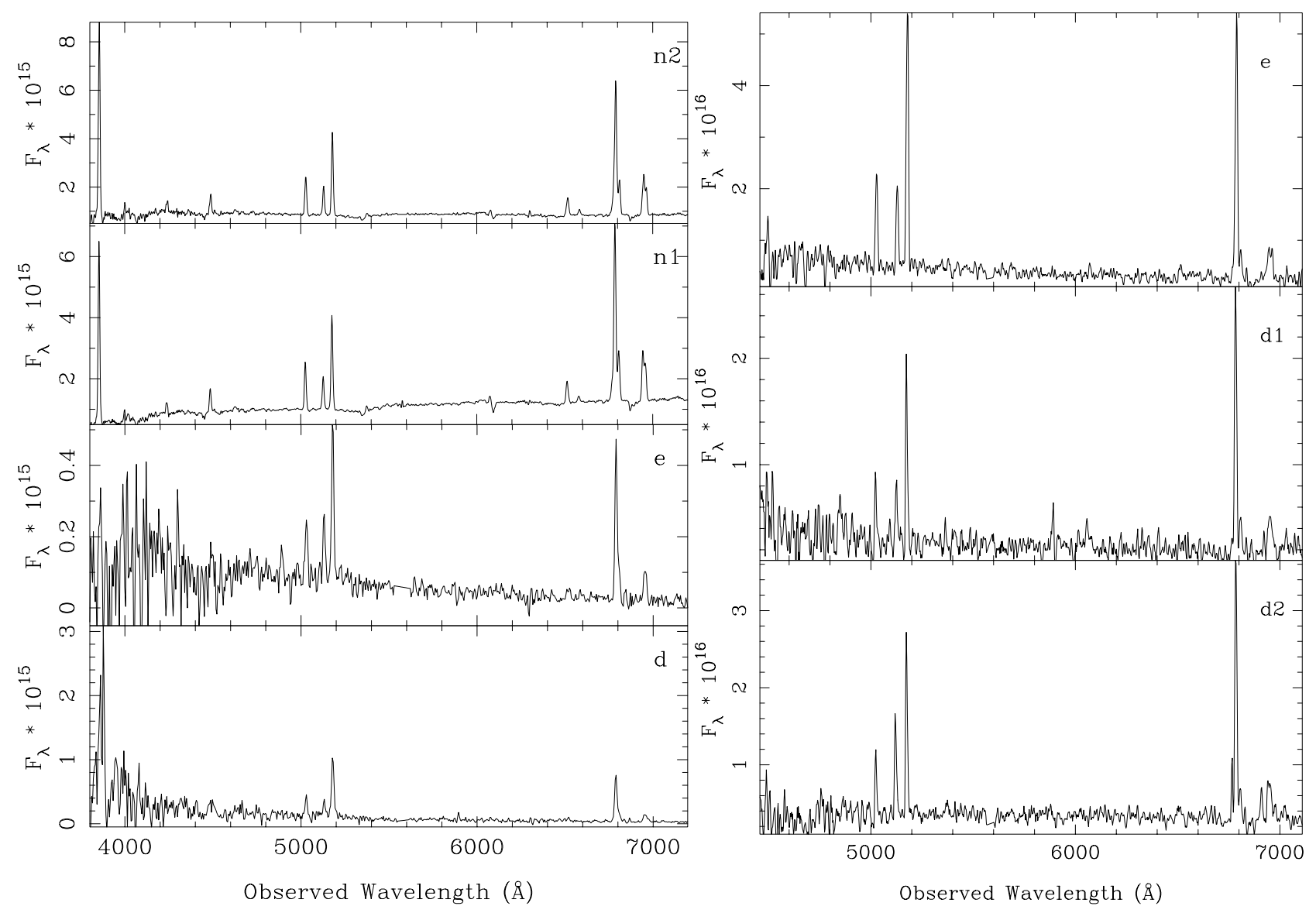

Fig. 6. 1D spectra extracted in the nucleus and in the d, e knots from the MPFS (left) and EFOSC 2 (right) data.

rotation curve for gas and stars. As mentioned above, [O III] and $\mathrm{H} \alpha$ show similar radial velocities, almost symmetrically distributed, with a $\Delta V_{\max } \sim 250 \mathrm{~km} \mathrm{~s}^{-1}$. Stars are well in agreement with the gaseous component only on the receding side, while poorly on the approaching side, with lower velocities by up to $-100 \mathrm{~km} \mathrm{~s}^{-1}$ with respect to the gas. From the analysis of the position angles of the rings, we obtained that the kinematical axes of gas and stars are not aligned, as expected, and are oriented at $\sim 30^{\circ}$ and $\sim 0^{\circ}$ respectively. Moreover, the kinematical axis of the gas is more stable in the receding side than in the approaching one, where the variations can reach $20^{\circ}$.

We have compared our rotation curve to that published by Moles et al. (2004), finding a general good agreement for the gaseous component. These authors used a long-slit spectrum taken close to the major axis, and obtained a curve with similar radial extension and velocities. Thanks to their higher spatial resolution they observed two rotating systems, which unfortunately are not visible in our data. However, we can assert that the stellar component shows rotation, at least in the region of the galaxy where the stellar kinematics could be measured.

We fitted the $\mathrm{H} \alpha$ curve with an analytic relation which assumes a spherical potential model with pure circular motion, $V_{\mathrm{c}}(R)=a R /\left(R^{2}+c_{0}^{2}\right)^{p / 2}$ (Bertola et al. 1991).

The nonlinear least-squares fit was stable, giving the following parameters: $a=515 \mathrm{~km} \mathrm{~s}^{-1}, c_{0}=3.8^{\prime \prime}$ and $p=1.27$. Using this relation, we estimated the total mass within a radius of $7^{\prime \prime}$ obtaining $\sim 7.3 \times 10^{10} M_{\odot}$.
The nuclear stellar velocity dispersion, $\sigma_{0}$, was also measured. First, the brightest central spectrum was considered, and then an average of central spectra within an aperture of $3^{\prime \prime}$. The results were similar. The estimate of $\sigma_{0}$ was again obtained with FXCOR by measuring the FWHM of the correlation peak, and then converting it into $\sigma_{0}$ following the recipes by Nelson $\&$ Whittle (1995). In detail, we convolved the template spectrum with Gaussian functions of increasing known $\sigma$, and we cross-correlated each convolved spectrum with the original one, obtaining a sort of $\sigma-F W H M$ empirical relation, which becomes flat for $\sigma<90 \mathrm{~km} \mathrm{~s}^{-1}$. The nuclear $\sigma_{0}$ was $\sim 170 \mathrm{~km} \mathrm{~s}^{-1}$, a value in agreement with the one measured by Moles et al. (2004), even if slightly lower.

Given the existence of a $\mathrm{Mg}-\sigma$ relation in early-type galaxies and spiral bulges, we measured the LICK/IDS system $\mathrm{Mg}$ and $\mathrm{Fe}$ indices in the center of the galaxy: $\mathrm{Mg}_{1}, \mathrm{Mg}_{2}, \mathrm{Mg}$ b, Fe5270, Fe5335, defined by Worthey et al. (1994), and we attempted a comparison with published values and relations. Since emission lines are sometimes present at the blue or red edges of these absorption features, the estimate of the continuum may be rather uncertain. Therefore, our measurements should be considered as rough estimates. $\mathrm{Mg}_{1}=0.08, \mathrm{Mg}_{2}=0.17, \mathrm{Mg} \mathrm{b}=3.6$ and $\langle\mathrm{Fe}\rangle=0.5(\mathrm{Fe} 5270+$ $\mathrm{Fe} 5335)=2.07$ are values typically observed in spiral bulges, and not in ellipticals or S0s (see e.g. Proctor \& Sansom 2002) at the $\sigma_{0}$ measured in Mkn 298. In other words, Mkn 298 is an outlier of the $\sigma$-Mg and $\sigma$-Fe relations, showing indices 


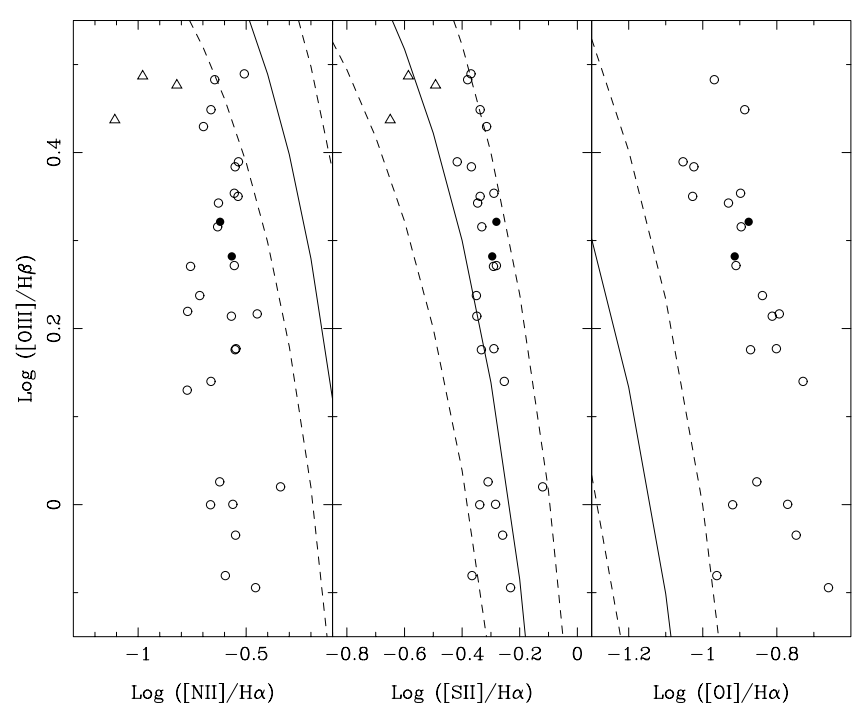

Fig. 7. VO diagnostic line ratios in Mkn 298. Circles: binned MPFS spectra in the nucleus. Dots: integrated MPFS spectra in the nucleus (N1, N2). Triangles: ESO spectra in the d, e knots. The solid line is the theoretical starburst border from Kewley et al. (2001), dashed lines are \pm 0.1 dex of these lines.

with lower strength than spheroidal galaxies. This effect can be caused either by age or by metallicity. On the other hand, the $[\mathrm{Mg} / \mathrm{Fe}]$ ratio is around 0.4 , indicating that the bulk of the nuclear stellar component is old probably formed on a timescale of 1-2 Gyr (Idiart et al. 1996). We can conclude that Mkn 298 does not fit the properties of the early-type galaxies because of the ongoing merger. Indeed, bursts of star formation induced by the strong interaction can effectively make the $\mathrm{Mg}$ and $\mathrm{Fe}$ indices strengths weaker because of the presence of bright, hot stars (Worthey \& Collobert 2003).

Similar results were obtained by Longhetti et al. (2000), who showed that shell galaxies, believed to be the product of a past strong interaction, deviate significantly from the $\sigma-\mathrm{Mg}$ and $\sigma$-Fe relations (see their Fig. 2). They proposed that this may be due either to the gravitational interaction which affects the velocity dispersion or to bursts of star formation that alter the metallicity indices, or to a combination of both.

\section{Emission line ratios}

Figure 7 shows the VO line ratios observed both in the nucleus and in the d, e knots: the lines dividing the theoretical starburst region from other types of excitation computed by Kewley et al. (2001) are overlaid. While in the d, e knots the line ratios are consistent with those expected in a starburst, the situation in the nucleus is more complex. In both the nuclear knots [N II] $\lambda 6583 / \mathrm{H} \alpha<0.3$ is typical of starburst regions, [S II] $\lambda \lambda 6716,6731 \sim 0.5$ may be fitted by starburst models, whereas $[\mathrm{OI}] / \mathrm{H} \alpha>0.1$ is too high for any starburst model. A similar behavior may be seen in all the individual spectra taken by the MPFS array in the nucleus. Figure 8 and Table 3 show that line ratios are remarkably constant over the whole galaxy (up to $5^{\prime \prime}$ from the center). Reddening by dust is low $(E(B-V)<0.50)$, with the exception of the regions on the western side $(E(B-V)>1)$, where it is due to the foreground dust lane.

\subsection{Chemical abundances}

As discussed in more detail in the next section, emission line ratios in the nucleus may imply the presence of an active nucleus. Chemical abundances in the nuclear regions were therefore computed using both the two calibrations proposed by Storchi-Bergmann et al. (1998, SSCK hereafter) for the NLR of active galaxies and the $R_{23}$ method (McGaugh 1991; Kobulnicky et al. 1999) and $p$ method (Pilyugin 2000, 2001) for abundances in H II regions of normal galaxies. We did not use [O III] $\lambda 4363$ due to the strong uncertainty in its measurement.

The SSCK calibrations are based on [N II] $\lambda \lambda$ 6548,6583/H $\alpha$ vs. [O III] $\lambda \lambda$ 4959,5007/H $\beta$ (Eq. (2) in SSCK) and [O II] $] \lambda 3727 /[\mathrm{O}$ III] $] \lambda \lambda 4959,5007$ (Eq. (3) in SSCK). Following the SSCK prescription we adopted the average of the two values, $12+\log (\mathrm{O} / \mathrm{H}) \sim 8.7\left(\mathrm{Z} / \mathrm{Z}_{\odot} \sim 0.7\right.$, assuming $\left.12+\log (\mathrm{O} / \mathrm{H})_{\odot}=8.87\right)$. To check these results we used the intensities given in Table 5 from Moles et al. (2004) and obtained consistent results, $12+\log (\mathrm{O} / \mathrm{H}) \sim 8.5\left(Z / Z_{\odot} \sim 0.6\right)$.

The $R_{23}$ and $p$ methods allow us to compute oxygen abundances from [O III $] \lambda \lambda 4959,5007 /[\mathrm{O}$ II $] \lambda 3727$. Both give as a result a lower abundance, $12+\log (\mathrm{O} / \mathrm{H}) \sim 8.3\left(Z / Z_{\odot} \sim 0.3\right)$.

For the d, e knots, Iglesias-Páramo et al. (2003) found $12+$ $\log (\mathrm{O} / \mathrm{H})=8.41\left(\mathrm{Z} / \mathrm{Z}_{\odot} \sim 0.35\right)$ using the $p$-method. A similar value, $12+\log (\mathrm{O} / \mathrm{H})=8.38\left(Z / Z_{\odot} \sim 0.33\right)$, is obtained from the emission line ratios that we measure in the e knot.

We therefore conclude that there is evidence for a sub-solar metallicity throughout the galaxy. The metallicity in the nucleus $\left(Z / Z_{\odot} \sim 0.5-0.7\right)$ may be somewhat higher than in the $\mathrm{d}$, e knots $\left(Z / Z_{\odot} \sim 0.3\right)$. We find no evidence supporting the very low metallicity $\left(Z / Z_{\odot} \sim 0.06\right)$ claimed by Moles et al. (2004).

Starting from these chemical abundances, we explored different models trying to fit the observed line spectra: pure photoionization models relative to an AGN and to a starburst, and composite models which account for both photoionization and shocks. In all the following models, $Z / Z_{\odot}$ was initially set to the value found here and then varied to give the best fit of the observed line ratios. The model which leads to the best fit of the line ratios will determine the nature of Mkn 298.

\subsection{Photoionization models}

As a first attempt to model the emission line ratios measured in the different regions, we assumed that they are produced by photoionization from either an active nucleus or stars (H II regions). To this aim, the photoionization code CLOUDY 94 (Ferland et al. 1998) was used.

In order to find the best-fit parameters from photoionization models, we ran a grid of models with different values of the ionization parameter and hydrogen density.

An attempt to use as the ionizing source a Kurucz continuum with temperatures up to $50000 \mathrm{~K}$ confirmed that in this case it is not possible to fit the $[\mathrm{OI}] / \mathrm{H} \alpha$ ratio. We then took 


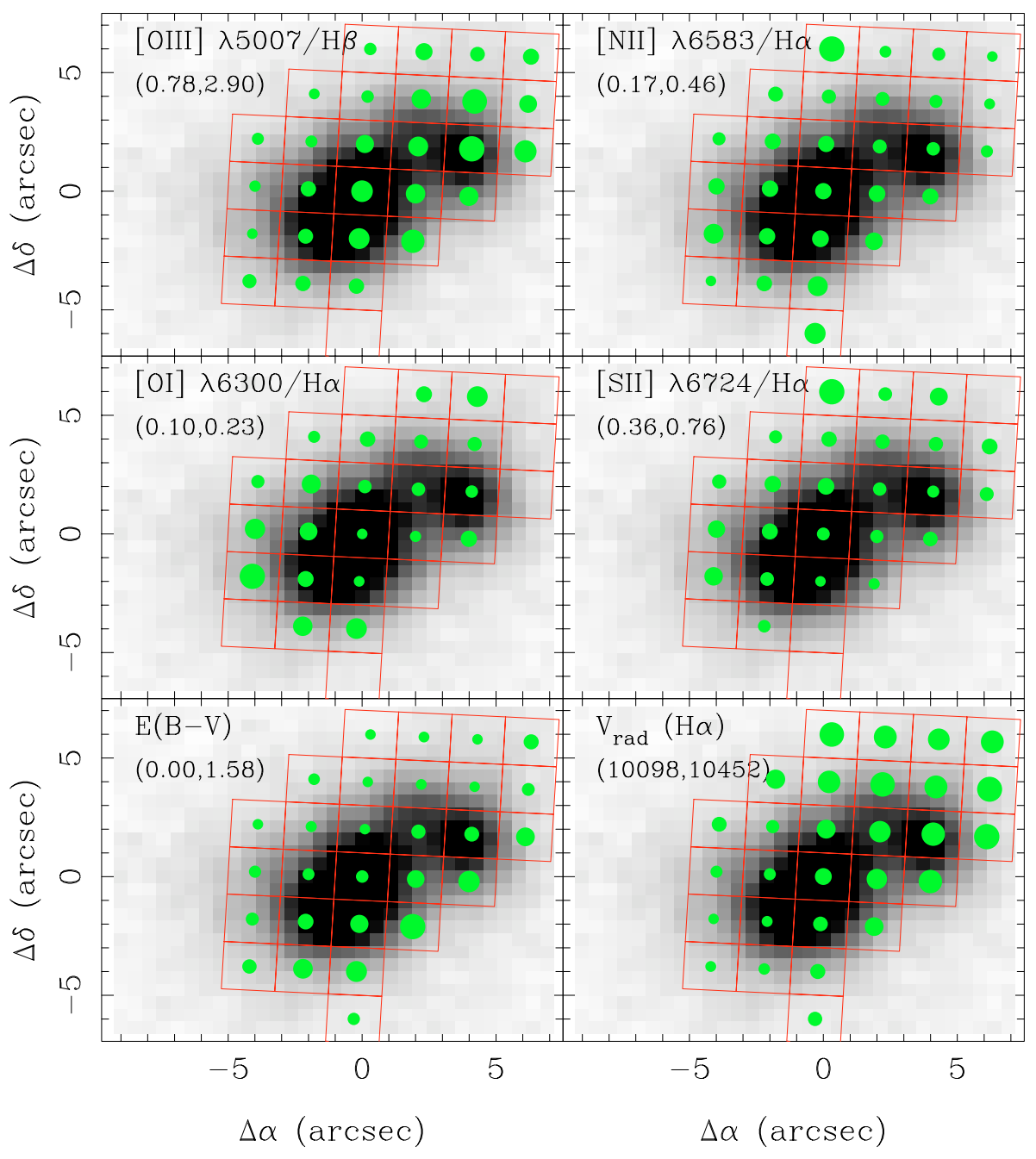

Fig. 8. Line ratios, dust extinction and $\mathrm{H} \alpha$ velocities from the rebinned MPFS spectra (R2) in the nucleus displayed as circles whose size is proportional to the measured values; the values in brackets are the minima and maxima. The $\mathrm{H} \alpha$ image is overlaid.

Table 3. Statistics of dereddened emission line ratios in the nucleus from the MPFS data (R1).

\begin{tabular}{lcccc}
\hline \hline & $\min$ & $\max$ & avg & $\sigma$ \\
\hline$[\mathrm{O}$ II $] \lambda 3727$ & 3.53 & 9.78 & 7.39 & 1.89 \\
{$[\mathrm{O}$ III $] \lambda 5007$} & 0.78 & 2.90 & 1.69 & 0.61 \\
{$[\mathrm{O} \mathrm{I}] \lambda 6300$} & 0.27 & 0.63 & 0.40 & 0.09 \\
$\mathrm{H} \alpha \lambda 6563$ & 2.18 & 2.80 & 2.76 & 0.13 \\
{$[\mathrm{~N} \mathrm{II}] \lambda 6583$} & 0.47 & 0.99 & 0.72 & 0.14 \\
{$[\mathrm{~S} \mathrm{II}] \lambda 6716$} & 0.56 & 1.08 & 0.79 & 0.12 \\
{$[\mathrm{~S} \mathrm{II}] \lambda 6731$} & 0.41 & 0.63 & 0.51 & 0.06 \\
{$[\mathrm{~S} \mathrm{II}] \lambda 6716+31$} & 1.01 & 1.65 & 1.30 & 0.16 \\
& & & & \\
$E(B-V)$ & 0.03 & 1.58 & 0.50 & 0.42 \\
\hline
\end{tabular}

as the ionizing source a power-law continuum (TABLE POWER LAW option in CLOUDY), $F_{v} \propto v^{-1.5}$.

Starting from the solar abundances given in CLOUDY ${ }^{3}$, abundances of all elements heavier than helium were scaled

\footnotetext{
${ }^{3}$ E.g. H: 1.00 , He: $0.1, \mathrm{C} / \mathrm{H}: 3.55 \times 10^{-4}$, N/H: $9.33 \times 10^{-5}, \mathrm{~S} / \mathrm{H}:$ $1.62 \times 10^{-5}, \mathrm{O} / \mathrm{H}: 7.41 \times 10^{-4}$.
}

as $Z / Z_{\odot}$, with the exception of nitrogen, which was scaled as $\left(Z / Z_{\odot}\right)^{2}$.

We considered a model acceptable if line ratios $(\mathrm{H} \beta=1)$ were consistent within $50 \%$ with the observed values (see Table 2 for results). In the nucleus, photoionization by stars is ruled out by the strong [O I] $\lambda 6300$ emission $([\mathrm{OI}] / \mathrm{H} \alpha \geq$ 0.1 ) which indicates ionization by a non-thermal source. A simultaneous fit of all the line fluxes, in particular of the low $[\mathrm{N} \mathrm{II}] \lambda 6583([\mathrm{~N} \mathrm{II}] / \mathrm{H} \alpha<0.3)$, is obtained with the following parameters: $Z / Z_{\odot}=0.5, n_{\mathrm{H}}=100 \mathrm{~cm}^{-3}, \log U=-3.4$.

In the regions located in the $\mathrm{d}$, e knots, the best fit was obtained using as the input ionizing source a Kurucz model atmosphere with $T=45000 \mathrm{~K}, \log n_{\mathrm{H}}=2 . \log U=-3, Z / Z_{\odot}=0.3$.

\subsection{Photoionization + shock models}

The presence of tidal tails strongly suggest that Mkn 298 could be a merging system leading to collisional processes. A more realistic model of the emission line ratios should then probably take into account the coupled effect of photoionizing radiation flux from an external source and of shocks. Therefore, we 


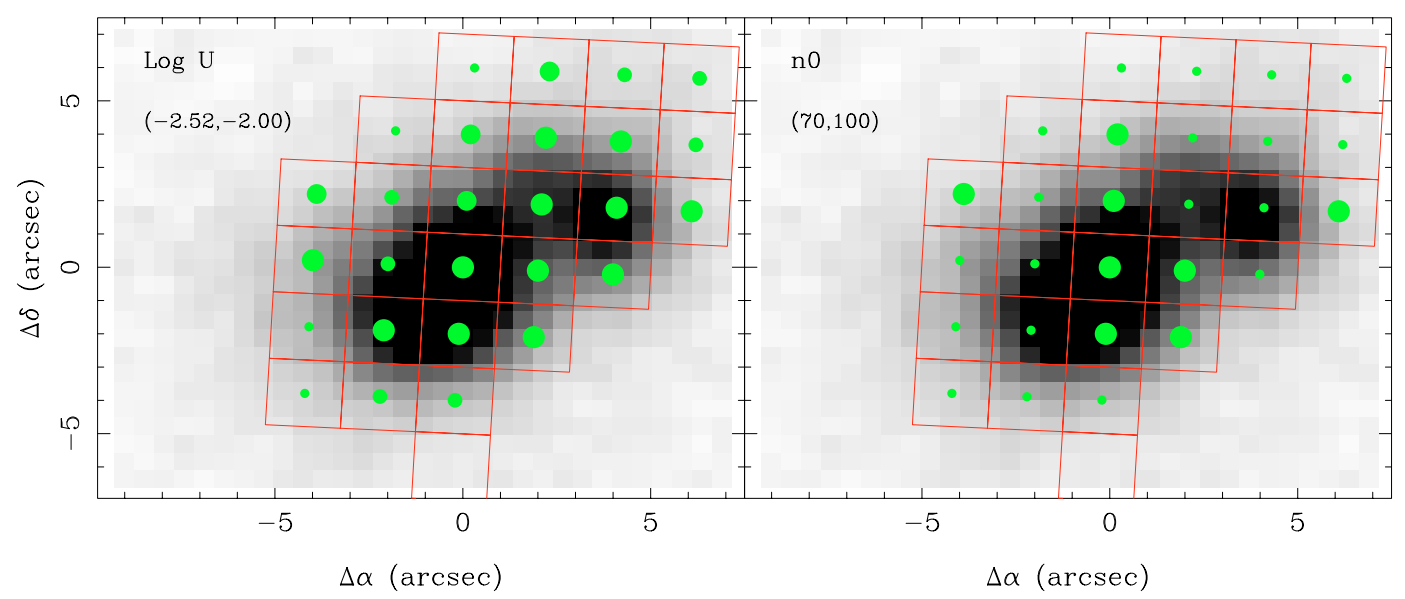

Fig. 9. Input parameters of the starburst+shock models which lead to the best fit of the line ratios in Fig. 8: $\log U($ left $), n_{0}($ right $)$.

have adopted the code SuMA (Viegas \& Contini 1994; Contini \& Viegas 2001a,b, and references therein) to model the spectra. SUMA allows one to calculate the spectra emitted from a gas which is heated and ionized by the coupled effect of a photoionizing radiation flux from an external source (active nucleus or stars) and of shocks. The input ionizing source is a power-law in the AGN case. Radiation from a stellar cluster is used for starbursts. The input parameters which refer to the shock are the shock velocity, $V_{\mathrm{s}}$, the preshock density, $n_{0}$, and the preshock magnetic field, $B_{0}$. The parameters of radiation are the radiation intensity $F_{\mathrm{h}}$ (in units of number of photons $\mathrm{cm}^{-2} \mathrm{~s}^{-1} \mathrm{eV}^{-1}$ at $1 \mathrm{Ryd}$ ) and the spectral index $\alpha$ in the case of a power-law, the age of the stellar population $t$ (in Myr) and the ionization parameter $U$ in the case of starbursts. Moreover, the input parameters include the geometrical thickness of the clouds, $D$, and the relative abundances of He, $\mathrm{C}, \mathrm{N}, \mathrm{O}, \mathrm{Ne}, \mathrm{Mg}, \mathrm{Si}, \mathrm{S}, \mathrm{Cl}, \mathrm{Ar}$, and Fe to H. A pre-shock magnetic field $B_{0}=10^{-4}$ Gauss was used for all models.

The shocked clouds are moving outward from the nucleus and radiation reaches the inner edge of the clouds opposite to the shock front.

A first set of models was computed by adding a shock component to the active nucleus. The best fit of the observed line ratios was obtained with the following parameters (Table 2): $V_{\mathrm{s}}=110 \mathrm{~km} \mathrm{~s}^{-1}, n_{0}=100 \mathrm{~cm}^{-3}, \log F_{\mathrm{H}}=7.15, D=0.36 \mathrm{pc}$. The abundances are nearly solar, with the exception of sulphur: $\mathrm{N} / \mathrm{H}=9 \times 10^{-5}, \mathrm{O} / \mathrm{H}=6.6 \times 10^{-4} ; \mathrm{S} / \mathrm{H}=1.1 \times 10^{-5}$,

In the case of a starburst + shock, the best fit was obtained with: $\mathrm{N} / \mathrm{H}=3.5 \times 10^{-5}, \mathrm{O} / \mathrm{H}=6.3 \times 10^{-4}, \mathrm{~S} / \mathrm{H}=5 \times 10^{-6}$ $\left(Z / Z_{\odot} \sim 0.6\right) ; \log U=-2.52, t=0.0-2.5 \mathrm{Myr}, D=0.5 \mathrm{pc}$, $V_{\mathrm{s}}=80 \mathrm{~km} \mathrm{~s}^{-1}, n_{0}=60 \mathrm{~cm}^{-3}$. Such values of the shock velocity and preshock density are typical of the bulk of the ionized gas in starburst galaxies (Viegas et al. 1999).

While in both cases the models provide a good fit of the observed line ratios, the flux from the active nucleus is extremely low, a factor $\sim 1000$ lower than what is derived e.g. in Seyfert $2 \mathrm{~s}, \log F_{\mathrm{H}}>11$ or in Liners, $\log F_{\mathrm{H}} \geq 8$ (Contini et al. 2003). Moreover, the [O III] $\lambda 4363 / \mathrm{H} \beta$ ratio is overpredicted by the AGN+shock models by a factor $\sim 10$.

We conclude that while the presence of an active nucleus cannot be excluded, it is not required in order to explain the observed line ratios, assuming that a shock component is also present. Ionization by a starburst, rather than by an active nucleus, appears to be also in better agreement with (i) the lack of a well-defined spatial trend of the line ratios with the distance from the putative active nucleus; and (ii) the morphology of the nucleus derived from the images in Sect. 3. Figure 9 displays the distribution of the ionization parameter $U$ and of the preshock density, $n_{0}$, which result from the best fit of the line ratios displayed in Fig. 8: we obtain $-2.00 \leq \log U \leq-2.52$, $70 \leq n_{0} \leq 100$, with higher preshock densities in N1. This confirms that the starburst+shock processes are spread over the whole nuclear region of Mkn 298.

The spectra of the d, e knots are all typical of starburst regions with $V_{\mathrm{s}}=90 \mathrm{~km} \mathrm{~s}^{-1}, n_{0}=50 \mathrm{~cm}^{-3}, \log U \sim-2.3$ and an age of the stellar cluster $t<2.5 \mathrm{Myr}$. The best fit was obtained with $\mathrm{N} / \mathrm{H}=1.5 \times 10^{-5}, \mathrm{O} / \mathrm{H}=3.3 \times 10^{-4} ; \mathrm{S} / \mathrm{H}=3.0 \times 10^{-6}$, which correspond to $Z / Z_{\odot} \sim 0.40$.

\section{Conclusions}

In this paper we have presented new imaging and integralfield spectroscopic data analysis of Mkn 298 to investigate its controversial nature. Using ground-based broad-band $R$ and HST band $V$ images we have performed a detailed analysis of the morphology of this galaxy, obtaining that Mkn 298 is an early-type spiral, likely a S0 galaxy $(\mathrm{B} / \mathrm{T} \sim 0.78)$, with a de Vaucouleurs bulge and without any evident nuclear pointlike source. We pointed out the presence of weak stellar shells around the galaxy. These shells, together with the bright and extended eastern tail, and the nuclear dust lane, strongly support the idea that Mkn 298 is the result of a major merger event. Ionized gas in Mkn 298 is distributed in two or maybe three main knots, visible in the $\mathrm{H} \alpha$-continuum subtracted image, and whose spectra mostly show the typical emission lines produced by thermal ionization. This gas is extended and oriented in the same direction as the dust lane, suggesting that these two features are likely to be connected. The kinematic investigation of $\mathrm{H} \alpha$ and [O III] velocity fields clearly show that the gas is rotating with a maximum deprojected velocity of $+250 \mathrm{~km} \mathrm{~s}^{-1}$ with respect to the systemic velocity of the galaxy. Therefore, the integral-field data confirm the previous 
hypothesis by Moles et al. (2004) that a disk dominated galaxy is the secondary component of the merger event.

The analysis of the emission line ratios shows that both AGN photoionization models and starburst+shock models with sub-solar metallicity $\left(Z / Z_{\odot} \leq 0.6\right)$ are able to fit the observed values. In particular the high $[\mathrm{OI}] / \mathrm{H} \alpha$, which could indicate the presence of an AGN, is also well reproduced by starburst+shock models, where photoionization is given by a young $(t<2.5 \mathrm{Myr})$ stellar population and shocks are produced in low velocity-density clouds $\left(V_{\mathrm{s}}=80 \mathrm{~km} \mathrm{~s}^{-1}, n_{0}=60 \mathrm{~cm}^{-3}\right)$. AGN+shock models are ruled by the $[\mathrm{O}$ III] $] \lambda 4363 / \mathrm{H} \beta$ ratio which is too high compared to the observed values and by the flux from the AGN which is unrealistically low.

Considering that emission line ratios are constant over all the regions where they are detected, with no well defined spatial trend, we conclude that the starburst+shock models provide a more likely possibility than an AG: merging would induce both collisional processes and star formation in the gas, thus producing the observed line ratios. No evidence was found for the very low metallicity claimed by Moles et al. (2004), $Z / Z_{\odot} \leq$ 0.1 . We also do not confirm the high $\operatorname{SFR}\left(\sim 90 M_{\odot} / \mathrm{yr}\right)$ found by Moles et al. (2004); we find instead that $\operatorname{SFR}(\mathrm{H} \alpha) \leq 10 M_{\odot} / \mathrm{yr}$. A comparable SFR was found taking the IRAS infrared luminosity at the position of Mkn 298, even if this value is very uncertain.

For the knots in the tidal tail $(\mathrm{d}, \mathrm{e})$, the presence of shocks is not compelling as both pure photoionization from stars and photoionization+shock models provide a good fit of the observed ratios. A sub-solar metallicity $\left(Z / Z_{\odot} \leq 0.4\right)$ is found in these regions as well.

Acknowledgements. We are grateful to the referee, P.-A. Duc, for his comments that improved the paper.

This paper is partly based on observations made with the NASA/ESA Hubble Space Telescope, obtained from the data archive at the Space Telescope Science Institute. STScI is operated by the Association of Universities for Research in Astronomy, Inc. under NASA contract NAS 5-26555. This research made use of the NASA/IPAC Extragalactic Database (NED) which is operated by the Jet Propulsion Laboratory, California Institute of Technology, under contract with the National Aeronautics and Space Administration. This research has also made use of the NASA/ IPAC Infrared Science Archive, which is operated by the Jet Propulsion Laboratory, California Institute of Technology, under contract with the National Aeronautics and Space Administration.

\section{References}

Balcells, M. 1997, ApJ, 486, L87

Balzano, V. A., \& Weedman, D. W. 1981, ApJ, 243, 756

Begeman, K. G. 1989, A\&A, 223, 47

Bertola, F., Bettoni, D., Danziger, J., et al. 1991, ApJ, 373, 369

Braine, J., Duc, P.-A., Lisenfeld, U., et al. 2001, A\&A, 378, 51

Carollo, C. M., Stiavelli, M., de Zeeuw, P. T., \& Mack, J. 1997, AJ, 114,2366

Ciroi, S., Contini, M., Rafanelli, P., \& Richter, G. M. 2003, A\&A, 400, 859

Contini, M., \& Viegas, S. M. 2001a, ApJS, 132, 211
Contini, M., \& Viegas, S. M. 2001b, ApJS, 137, 75

Contini, M., Viegas, S. M., \& Campos, P. E. 2003, MNRAS, 346, 37

Cutri, R. M., et al. 2003, http://www.ipac. caltech.edu/2mass

De Vaucouleurs, G., De Vaucouleurs, A., Corwin, J., et al. 1991, Third reference catalogue of bright galaxies, version 39

Ferland, G. J., Korista, K. T., Verner, D. A., et al. 1998, PASP, 110, 761

Gavazzi, G., \& Boselli, A. 1996, ApL\&C, 35, 1

Georgakakis, A., Forbes, D. A., \& Norris, R. P. 2000, MNRAS, 318, 124

Hernquist, L., \& Spergel, D. N. 1992, ApJ, 1, 399, L117

Hibbard, J. E., \& van Gorkom, J. H. 1996, AJ, 111, 655

Hopkins, A. M., Miller, C. J., Nichol, R. C., et al. 2003, ApJ, 599, 971

Huchra, J. P. 1977, ApJS, 35, 171

Idiart, T. P., de Freitas Pacheco, J. A., \& Costa, R. D. D. 1996, AJ, 112,2541

Iglesias-Páramo, J., van Driel, W., Duc, P.-A., et al. 2003, A\&A, 406, 453

Israel, F. P., Seielstad, G. A., \& Berge, G. L. 1988, A\&A, 189, 7

McGaugh, S. S. 1991, ApJ, 380, 140

Kennicutt, R. C. 1998, ARA\&A, 36, 189

Kewley, L. J., Dopita, M. A., Sutherland, R. S., et al. 2001, ApJ, 556, 121

Kobulnicky, H. A., Kennicutt, R. C., \& Pizagno, J. L. 1999, ApJ, 514, 544

Koski, A. T. 1978, ApJ, 223, 56

Metik, L. P., \& Pronik, I. I. 1982, Astrophys., 17, 333

Longhetti, M., Bressan, A., Chiosi, C., \& Rampazzo, R. 2000, A\&A, 353, 917

Malkan, M. A., Gorjian, V., \& Tam, R. 1998, ApJS, 117, 25

Moles, M., Bettoni, D., Fasano, G., et al. 2004, A\&A, 418, 495

Morgan, H. L., \& Edmunds, M. G. 2003, MNRAS, 343, 427

Nelson, C. H., \& Whittle, M. 1995, ApJS, 99, 67

Pilyugin, L. 2000, A\&A, 362, 325

Pilyugin, L. 2001, A\&A, 369, 594

Proctor, R. N., \& Sansom, A. E. 2002, MNRAS, 333, 517

Rafanelli, P., Afanasiev, V., Birkle, K., et al. 1999, Mem. Soc. Astron. It., 70,81

Storchi-Bergmann, T., Schmitt, H. R., Calzetti, D., \& Kinney, A. L. 1998, AJ, 115, 909

Simard, L., Willmer, C. N. A., Vogt, N. P., et al. 2002, ApJS, 142, 1

Stockton, A. 1972, ApJ, 173, 247

Takamiya, M., \& Kron, G. 1995, AJ, 110, 1083

Tonry, J., \& Davis, M. 1979, AJ, 84, 1511

Turnbull, A. J., Bridges, T. J., \& Carter, D. 1999, MNRAS, 307, 967

van Zee, L., Salzer, J. J., Haynes, M. P., O’Donoghue, A. A., \& Balonek, T. J. 1998, AJ, 116, 2805

Veilleux, S., \& Osterbrock, D. E. 1987, ApJS, 63, 295

Verdes-Montenegro, L., Del Olmo, A., Iglesias-Páramo, J. I., et al. 2002, A\&A, 396, 815

Viegas, S. M., \& Contini, M. 1994, ApJ, 428, 113

Viegas, S. M., Contini, M., \& Contini, T. 1999, A\&A, 347, 112

Viegas-Aldrovandi, S. M., \& Gruenwald, R. B. 1990, ApJ, 360, 474

Worthey, G., Faber, S. M., Gonzalez, J. J., \& Burstein, D. 1994, ApJS, 94, 687

Worthey, G., \& Collobert, M. 2003, ApJ, 586, 17

Zezas, A., Fabbiano, G., Rots, H., \& Murray, S. S. 2002, ApJ, 577, 10

Zwicky, F., \& Herzog, E. 1963, Catalogue of Galaxies and of Clusters of Galaxies, Volume II (Pasadena: California Institute of Technology) 